MATHEMATICS OF COMPUTATION

Volume 66, Number 218, April 1997, Pages 743-761

S $0025-5718(97) 00832-6$

\title{
ACCELERATED POLYNOMIAL APPROXIMATION OF FINITE ORDER ENTIRE FUNCTIONS BY GROWTH REDUCTION
}

\author{
JÜRGEN MÜLLER
}

\begin{abstract}
Let $f$ be an entire function of positive order and finite type. The subject of this note is the convergence acceleration of polynomial approximants of $f$ by incorporating information about the growth of $f(z)$ for $z \rightarrow \infty$. We consider "near polynomial approximation" on a compact plane set $K$, which should be thought of as a circle or a real interval. Our aim is to find sequences $\left(f_{n}\right)_{n}$ of functions which are the product of a polynomial of degree $\leq n$ and an "easy computable" second factor and such that $\left(f_{n}\right)_{n}$ converges essentially faster to $f$ on $K$ than the sequence $\left(P_{n}^{*}\right)_{n}$ of best approximating polynomials of degree $\leq n$. The resulting method, which we call Reduced Growth method ( $R G$-method) is introduced in Section 2. In Section 5, numerical examples of the $R G$-method applied to the complex error function and to Bessel functions are given.
\end{abstract}

\section{INTRODUCTION}

Let $K$ be a compact subset of the complex plane $\mathbb{C}$. To avoid technical difficulties we will assume, if not otherwise stated, that $K$ and $\mathbb{C} \backslash K$ are connected and $K$ does not consist of a single point. (In fact we are mainly interested in the cases of $K$ being a disk or an interval.) Moreover, by $\hat{\mathbb{C}}:=\mathbb{C} \cup\{\infty\}$ we denote the extended complex plane and we set

$$
\boldsymbol{\Delta}_{r}:=\{z \in \mathbb{C}:|z| \leq r\}, \quad \boldsymbol{\Delta}:=\boldsymbol{\Delta}_{1} .
$$

According to the Riemann mapping theorem there exists a uniquely determined conformal mapping $\psi: \hat{\mathbb{C}} \backslash \boldsymbol{\Delta} \rightarrow \hat{\mathbb{C}} \backslash K$ such that

$$
\psi(w)=c w+\sum_{\nu=0}^{\infty} c_{\nu} w^{-\nu} \quad(|w|>1)
$$

and $c=c(K)>0$. In this case, $c(K)$ is the logarithmic capacity of $K$.

In the sequel we will consider entire functions of finite order $\rho>0$ and type $\tau$, i.e., we assume

and

$$
\rho=\rho_{f}=\limsup _{r \rightarrow \infty} \frac{\log \log M(r, f)}{\log r} \in(0, \infty)
$$

$$
\tau=\tau_{f}=\limsup _{r \rightarrow \infty} \frac{\log M(r, f)}{r^{\rho}}<\infty
$$

Received by the editor October 16, 1995 and, in revised form, April 1, 1996.

1991 Mathematics Subject Classification. Primary 65B99; Secondary 30D10.

(C)1997 American Mathematical Society 
where $M(r, f):=\|f\|_{\Delta_{r}}$. Let $\Pi_{n}$ be the set of polynomials of degree $\leq n$ and let

$$
E_{n}(f, K):=\inf _{P \in \Pi_{n}}\|f-P\|_{K},
$$

with $\|\varphi\|_{K}:=\sup _{z \in K}|\varphi(z)|$, denote the error of best polynomial approximation of $f$ on $K$. The following result seems to be first established by Batyrev and may be found, under more general assumptions on $K$, in [31] or [24].

Theorem 1. Let $K \subset \mathbb{C}$ be a compact set as above, and let $f: K \rightarrow \mathbb{C}$ be a function. Then $f$ is the restriction on $K$ of an entire function of order $\rho$ and type $\tau$ if and only if

$$
\limsup _{n \rightarrow \infty} n^{1 / \rho} E_{n}(f, K)^{1 / n}=c(K)(e \rho \tau)^{1 / \rho} .
$$

A sequence $\left(P_{n}\right)$ with $P_{n} \in \Pi_{n}$ for all $n \in \mathbb{N}$ is called maximally convergent on $K$ to $f$ if the asymptotic rate of best polynomial approximation is realized by $\left(P_{n}\right)$, that is

$$
\limsup _{n \rightarrow \infty} n^{1 / \rho}\left\|f-P_{n}\right\|_{K}^{1 / n}=c(K)(e \rho \tau)^{1 / \rho} .
$$

Besides the polynomials $P_{n}^{*}$ of best approximation given by

$$
\left\|f-P_{n}^{*}\right\|_{K}=\inf _{P \in \Pi_{n}}\|f-P\|_{K}
$$

the computation of which is rather expensive, mainly two types of maximally convergent sequences $\left(P_{n}\right)$ are considered: polynomial interpolants in equidistributed nodes and Faber expansions. We will briefly recall these two kinds of polynomial approximants.

1. Let $\left(z_{k}^{(n)}\right)_{n \in \mathbb{N}_{0}, k=0, \ldots, n}$ be an infinite matrix (the node matrix) such that $z_{k}^{(n)} \in K$ for $n \in \mathbb{N}_{0}$ and $k=0, \ldots, n$ and let

$$
\omega_{n}(z)=\prod_{k=0}^{n}\left(z-z_{k}^{(n)}\right) \quad\left(n \in \mathbb{N}_{0}\right) .
$$

If $\Gamma_{r}=\psi\left(\partial \boldsymbol{\Delta}_{r}\right)$ is a level curve of $\psi^{-1}$ for some $r>1$, then the (uniquely determined) polynomial interpolant $L_{n}(f) \in \Pi_{n}$ to $f$ with respect to the nodes $z_{k}^{(n)}$ may be expressed by the Hermite interpolation formula

$$
L_{n}(z)=L_{n}(f)(z)=\frac{1}{2 \pi i} \int_{\Gamma_{r}} \frac{\omega_{n}(t)-\omega_{n}(z)}{t-z} \frac{f(t)}{\omega_{n}(t)} d t \quad(z \in K) .
$$

The nodes $z_{k}^{(n)}$ are called equidistributed on $K$, if

$$
\left\|\omega_{n}\right\|_{K}^{1 /(n+1)} \rightarrow c(K) \quad(n \rightarrow \infty) .
$$

We refer to [9], where examples of equidistributed nodes are given. In particular, if $K=\boldsymbol{\Delta}_{r}$ for some $r>0$ and if $z_{k}^{(n)}=0$ for all $k$ and $n$, then we obtain

$$
L_{n}(f)=S_{n}(f),
$$

where $S_{n}(f)$ is the $n$-th partial sum of the Taylor expansion of $f$ around the origin.

2. Let $K$ and $\psi$ be as above. The $n$-th Faber polynomial $F_{n}=F_{n, K}$ with respect to $K$ may be defined by

$$
\frac{\psi^{\prime}(w)}{\psi(w)-z}=\sum_{n=0}^{\infty} \frac{F_{n}(z)}{w^{n+1}} \quad(z \in K)
$$


By a well-known result of Kövari and Pommerenke (see [17]), there exist constants $A>0$ and $\alpha<0.5$ such that for every $f$ holomorphic on $K$ (i.e. holomorphic in a neighbourhood of $K$ )

$$
\left\|f-T_{n}(f)\right\|_{K} \leq A n^{\alpha} E_{n}(f, K),
$$

where $T_{n}(f)$ denotes the $n$-th partial sum of the Faber expansion of $f$, that is

$$
T_{n}(f)=T_{n, K}(f)=\sum_{k=0}^{n} a_{k}(f) F_{k}
$$

with

$$
a_{k}(f)=\frac{1}{2 \pi i} \int_{|w|=1} \frac{f(\psi(w))}{w^{n+1}} d w .
$$

From (5) it follows that $\left(T_{n}(f)\right)$ converges maximally on $K$ to $f$.

In particular, for $K=\boldsymbol{\Delta}_{r}$ we have $T_{n, \boldsymbol{\Delta}_{r}}(f)=S_{n}(f)$, and therefore Theorem 1 implies

$$
\limsup _{n \rightarrow \infty} n^{1 / \rho}\left\|f-S_{n}(f)\right\|_{\Delta_{r}}^{1 / n}=r(e \rho \tau)^{1 / \rho}
$$

(note that $c\left(\boldsymbol{\Delta}_{r}\right)=r$ ). Moreover, for $K=[-1,1]$, the $n$-th partial sum of the Faber expansion of $f$ equals the $n$-th partial sum of the Tschebyscheff expansion of $f$. Since $c([-1,1])=1 / 2$, Theorem 1 yields

$$
\limsup _{n \rightarrow \infty} n^{1 / \rho}\left\|f-T_{n}(f)\right\|_{[-1,1]}^{1 / n}=\frac{1}{2}(e \rho \tau)^{1 / \rho} .
$$

Two facts should be emphasized: On the one hand, the asymptotic rate of best polynomial approximation on $K$ of an entire function of finite order is determined by the growth parameters order and type of $f$, and, on the other hand, information on the growth of $f$, which is often available from theoretical investigations, cannot be used to improve the rate of convergence for polynomial approximation of $f$.

The basic idea in the sequel is the following: Use information about the growth of $f$ to modify the function $f$ in such a way that the modified $\tilde{f}$ is "better" approximable on $K$ by polynomials than $f$ itself, and then recover $f$ from an approximation of $\tilde{f}$.

\section{The RG-METHOD}

We first want to describe the underlying idea in the special case of $K$ being the closed unit disk and the partial sums $S_{n}$ of the Taylor expansion around the origin as approximating polynomials.

Let, for an arbitrary power series

$$
g(z)=\sum_{\nu=0}^{\infty} g_{\nu} z^{\nu}
$$

around the origin,

$$
S_{n}(g)(z)=\sum_{\nu=0}^{n} g_{\nu} z^{\nu}
$$


denote the $n$-th Taylor section of $g$. For $g$ being holomorphic on $\boldsymbol{\Delta}_{r}$ for some $r>1$, we obtain from the Cauchy integral formula

$$
\left\|g-S_{n}(g)\right\|_{\Delta} \leq \frac{M(r, g)}{r^{n}(r-1)} .
$$

Thus, if $f$ is an entire function of order $\rho$ and type $\tau<\infty$, and if $\left(r_{n}\right)$ is an arbitrary sequence with $1<r_{n} \rightarrow \infty$, we get

$$
\limsup _{n \rightarrow \infty} n^{1 / \rho}\left\|f-S_{n}(f)\right\|_{\Delta}^{1 / n} \leq \limsup _{n \rightarrow \infty} \frac{n^{1 / \rho} M\left(r_{n}, f\right)^{1 / n}}{r_{n}} .
$$

If $\tau>0$ and if we take

$$
r_{n}=\left(\frac{n}{\tau \rho}\right)^{1 / \rho}
$$

then we find

$$
\limsup _{n \rightarrow \infty} \frac{n^{1 / \rho} M\left(r_{n}, f\right)^{1 / n}}{r_{n}} \leq(e \rho \tau)^{1 / \rho} .
$$

Since $c(\boldsymbol{\Delta})=1$, this implies in particular that $S_{n}(f)$ converges to $f$ maximally on $\Delta$.

Now the simple idea is the following: We replace in the above estimates $f$ by $f \varphi_{n}$, where $\left(\varphi_{n}\right)_{n}$ is a sequence of functions such that $\varphi_{n}$ is holomorphic on $\boldsymbol{\Delta}_{r_{n}}$ for all $n \in \mathbb{N}$. With $\varphi:=\left(r_{n}, \varphi_{n}\right)_{n}$ and

$$
\mu(\varphi):=\limsup _{n \rightarrow \infty} \frac{n^{1 / \rho} M\left(r_{n}, f \varphi_{n}\right)^{1 / n}}{r_{n}}
$$

we get as above

$$
\limsup _{n \rightarrow \infty} n^{1 / \rho}\left\|f \varphi_{n}-S_{n}\left(f \varphi_{n}\right)\right\|_{\Delta}^{1 / n} \leq \mu(\varphi) .
$$

If $\mu(\varphi)<(e \rho \tau)^{1 / \rho}$ and if $\left|\varphi_{n}\right|^{1 / n}$ converges to 1 uniformly on $\boldsymbol{\Delta}$, then the sequence $\left(\varphi_{n}^{-1} S_{n}\left(f \varphi_{n}\right)\right)_{n}$ converges asymptotically by the factor $\left(\mu(\varphi) /(e \rho \tau)^{1 / \rho}\right)^{n}$ faster to $f$ than maximally convergent polynomial sequences.

Using (2), one can prove in a similar way (cf. [21]) the following more general result.

Theorem 2. Let $K \subset \mathbb{C}$ be a compact set such that $\hat{\mathbb{C}} \backslash K$ is simply connected, $K$ not a single point, and let $f$ be an entire function of order $\rho \in(0, \infty)$ and type $\tau<\infty$.

Suppose further that $\varphi:=\left(r_{n}, \varphi_{n}\right)_{n}$ is a sequence such that $0<r_{n} \rightarrow \infty$ for $n \rightarrow \infty$ and $\varphi_{n}$ is a function which is holomorphic on $\boldsymbol{\Delta}_{r_{n}} \cup K$.

If $\left(z_{k}^{(n)}\right)_{n \in \mathbb{N}_{0}, k=0, \ldots, n}$ is a matrix of equidistributed nodes on $K$, then

$$
\limsup _{n \rightarrow \infty} n^{1 / \rho}\left\|f \varphi_{n}-L_{n}\left(f \varphi_{n}\right)\right\|_{K}^{1 / n} \leq c(K) \mu(\varphi) .
$$

The estimates (1) and (10) suggest the following idea for an algorithm:

1. Search for a sequence $\varphi=\left(r_{n}, \varphi_{n}\right)_{n}$ as in Theorem 2 such that

$$
\mu(\varphi)<(e \rho \tau)^{1 / \rho}
$$

and $\left|\varphi_{n}\right|^{1 / n} \rightarrow 1$ locally uniformly on $\mathbb{C}$.

2. Compute an approximating polynomial $P_{n}=P_{n}\left(f \varphi_{n}, K\right)$ of $f \varphi_{n}$.

3. Take $\varphi_{n}^{-1} \cdot P_{n}\left(f \varphi_{n}\right)$ as approximation of $f$. 
In the sequel, we will refer to this method as the Reduced Growth method ( $R G$ method).

Looking at step 1 of our proposed algorithm, two questions arise:

(i) Let $\Phi$ be the set of all sequences $\varphi=\left(r_{n}, \varphi_{n}\right)_{n}$ as in Theorem 2 with $\left|\varphi_{n}\right|^{1 / n} \rightarrow$ 1 locally uniformly on $\mathbb{C}$. Can we determine

$$
\underline{m}=\underline{m}_{f}:=\inf _{\varphi \in \Phi} \mu(\varphi) \quad ?
$$

(ii) If so, how can we find "easy computable" sequences $\varphi \in \Phi$ such that $\mu(\varphi) \approx$ $\underline{m}$ ?

To answer these questions we have to throw a closer look at $f$. The indicator function $h=h_{f}:[-\pi, \pi] \rightarrow \mathbb{R}$ of $f$ is defined by

$$
h_{f}(\vartheta):=\limsup _{r \rightarrow \infty} \frac{\log \left|f\left(r e^{i \vartheta}\right)\right|}{r^{\rho}} \quad(\vartheta \in[-\pi, \pi]) .
$$

This function reflects the asymptotic growth behaviour of $f$ at infinity. In particular, from leading terms of asymptotic expansions we may obtain $h_{f}$. In the following we assume $h_{f}$ to be known.

From the definition it follows directly that $h_{f}(\vartheta) \leq \tau$ for all $\vartheta$. Moreover, it is a well-known fact (see for example [20], p. $275_{2}$ f) that actually

$$
-\tau \leq h_{f}(\vartheta) \leq \tau \quad(\vartheta \in[-\pi, \pi])
$$

and that $h_{f}$ is continuous (with $h_{f}(-\pi)=h_{f}(\pi)$ ). The crucial role in our game is played by

$$
\underline{\tau}=\underline{\tau}_{f}:=\frac{1}{2 \pi} \int_{-\pi}^{\pi} h_{f}(\vartheta) d \vartheta \text {. }
$$

The value $\underline{\tau}$ is intimately related to the number of zeros of $f$, as may be seen from classical results on entire functions found for example in [18], Chapter 4. Let $n_{f}(r)$ denote the number of zeros of $f$ in $\boldsymbol{\Delta}_{r}$ (counted with multiplicity). Then, if $f$ is of completely regular growth (for a definition see [18], Chapter 3, or [2], Definition 1.5.9), we have

$$
\lim _{r \rightarrow \infty} \frac{n_{f}(r)}{r^{\rho}}=\rho \underline{\tau} .
$$

From a result of Steinmetz ([27]) it may be seen ([21]) that in particular all entire functions which are solutions of $m$-th order homogeneous linear differential equations with rational coefficient functions are of completely regular growth.

A most simple conclusion from the above cited results is the fact that always $\underline{\tau} \geq 0$ (and thus $\underline{\tau} \in[0, \tau]$ ). The following result gives an answer to question (i).

Lemma 3. Let $f$ be an entire function of order $\rho>0$ and of completely regular growth. Then we have

$$
\underline{m}=(e \rho \underline{\tau})^{1 / \rho} .
$$

For a proof of the inequality $\geq$ we refer to [21]. The converse inequality $\leq$ follows for example from Theorem 6 below.

We now turn to the question (ii) of how to find sequences $\varphi \in \Phi$ such that

$$
\mu(\varphi) \approx(e \rho \underline{\tau})^{1 / \rho}
$$


and such that the functions $\varphi_{n}$ are "easy computable". We restrict ourselves to sequences $\left(\varphi_{n}\right)$ of the form $\varphi_{n}=e^{-R_{n}}$, where the $R_{n}$ are polynomials. In [21], however, also sequences $\left(\varphi_{n}\right)$ of rational functions are applied.

For given $h_{f}$ we consider a polynomial $Q$ such that $Q(0)=0$, and we set

$$
\tau(Q)=\tau_{f}(Q):=\max _{\vartheta}\left(h_{f}(\vartheta)-\operatorname{Re} Q\left(e^{i \vartheta}\right)\right) .
$$

Since $\operatorname{Re} Q$ is subharmonic on $\boldsymbol{\Delta}$, we find

$$
\frac{1}{2 \pi} \int_{-\pi}^{\pi}\left[h_{f}(\vartheta)-\operatorname{Re} Q\left(e^{i \vartheta}\right)\right] d \vartheta \geq \underline{\tau}-\operatorname{Re} Q(0)=\underline{\tau}
$$

and thus

$$
\tau(Q) \geq \underline{\tau} .
$$

In the sequel we will always assume $\tau(Q)>0$. We define

$$
r_{n}:=\left(\frac{n}{\tau(Q) \rho}\right)^{1 / \rho}
$$

and

$$
\varphi_{n}(z)=\varphi_{n, Q}(z):=\exp \left(-r_{n}^{\rho} Q\left(z / r_{n}\right)\right) \quad(z \in \mathbb{C}) .
$$

Then it is easily seen that $\varphi=\left(r_{n}, \varphi_{n}\right)_{n} \in \Phi$ and one can prove

Lemma 4. Let $\left(r_{n}\right)$ and $\left(\varphi_{n}\right)$ be defined by (14) and (15). Then we have

$$
\mu(\varphi) \leq(e \rho \tau(Q))^{1 / \rho} .
$$

Proof. For every $n \in \mathbb{N}$ we have $r_{n}^{-\rho} \log \left|\varphi_{n}\left(r_{n} e^{i \vartheta}\right)\right|=-\operatorname{Re} Q\left(e^{i \vartheta}\right)$ and thus

$$
\log \frac{M\left(r_{n}, f \varphi_{n}\right)}{r_{n}^{\rho}} \leq \max _{\vartheta}\left[\frac{\log \left|f\left(r_{n} e^{i \vartheta}\right)\right|}{r_{n}^{\rho}}-h_{f}(\vartheta)\right]+\max _{\vartheta}\left[h_{f}(\vartheta)-\operatorname{Re} Q\left(e^{i \vartheta}\right)\right] .
$$

From Theorem 28, Chapter I, of [18] one can deduce

$$
\limsup _{n \rightarrow \infty} \max _{\vartheta}\left[\frac{\log \left|f\left(r_{n} e^{i \vartheta}\right)\right|}{r_{n}^{\rho}}-h_{f}(\vartheta)\right] \leq 0
$$

and therefore

$$
\limsup _{n \rightarrow \infty} \frac{\log M\left(r_{n}, f \varphi_{n}\right)}{r_{n}^{\rho}} \leq \tau(Q) .
$$

According to (14) we find

$$
\mu(\varphi)=\limsup _{n \rightarrow \infty} \frac{n^{1 / \rho}\left(M\left(r_{n}, f \varphi_{n}\right)^{1 / r_{n}^{\rho}}\right)^{r_{n}^{\rho} / n}}{r_{n}} \leq(e \rho \tau(Q))^{1 / \rho} .
$$

The following is the main result of Section 2 .

Theorem 5. Let $K \subset \mathbb{C}$ be a compact set such that $\hat{\mathbb{C}} \backslash K$ is simply connected, $K$ not a single point and let $Q$ be a polynomial with $Q(0)=0$. Suppose further that $f$ is an entire function of order $\rho \in(0, \infty)$ and type $\tau<\infty$ and that $\left(\varphi_{n}\right)$ is given by (15).

1. If $\left(z_{k}^{(n)}\right)_{n \in \mathbb{N}_{0}, k=0, \ldots, n}$ is a matrix of equidistributed nodes on $K$, then

$$
\limsup _{n \rightarrow \infty} n^{1 / \rho}\left\|f-\varphi_{n}^{-1} L_{n}\left(f \varphi_{n}\right)\right\|_{K}^{1 / n} \leq c(K)(e \rho \tau(Q))^{1 / \rho} .
$$


2. Let $\mathcal{E}$ denote the set of all entire functions. If $\left(T_{n}\right)$ is a sequence of operators $T_{n}: \mathcal{E} \rightarrow \Pi_{n}$ such that there exist constants $A, \alpha>0$ with

$$
\left\|g-T_{n}(g)\right\|_{K} \leq A n^{\alpha} E_{n}(g, K)
$$

for all $g \in \mathcal{E}$, then

$$
\limsup _{n \rightarrow \infty} n^{1 / \rho}\left\|f-\varphi_{n}^{-1} T_{n}\left(f \varphi_{n}\right)\right\|_{K}^{1 / n} \leq c(K)(e \rho \tau(Q))^{1 / \rho} .
$$

Proof. 1. Since $\left|\varphi_{n}\right|^{-1 / n} \rightarrow 1$ uniformly on $K$, the assertion of 1 . follows directly from Theorem 2 and Lemma 4.

2. Let $L_{n}\left(f \varphi_{n}\right)$ denote the $n$-th polynomial interpolant to $f \varphi_{n}$ with respect to the system of the Fekete nodes of $K$. Since the Fekete nodes are equidistributed on $K$ (see for example [9]), by Theorem 2 and Lemma 4 we find

$$
\begin{gathered}
\limsup _{n \rightarrow \infty} n^{1 / \rho}\left\|f \varphi_{n}-T_{n}\left(f \varphi_{n}\right)\right\|_{K}^{1 / n}=\limsup _{n \rightarrow \infty} n^{1 / \rho} E_{n}\left(f \varphi_{n}, K\right)^{1 / n} \\
\leq \limsup _{n \rightarrow \infty} n^{1 / \rho}\left\|f \varphi_{n}-L_{n}\left(f \varphi_{n}\right)\right\|_{K}^{1 / n} \leq c(K)(e \rho \tau(Q))^{1 / \rho} .
\end{gathered}
$$

Since $\left|\varphi_{n}\right|^{-1 / n} \rightarrow 1$ uniformly on $K$, we obtain our assertion.

Remark. Obviously, condition (18) is satisfied by the sequence of best approximation operators, that is, $T_{n}(g)$ is defined by

$$
\left\|g-T_{n}(g)\right\|_{K}=\inf _{P \in \Pi_{n}}\|g-P\|_{K} \quad(g \in H(K)) .
$$

Moreover, by the above cited result of Kövari and Pommerenke (5), the same is true for the sequence $\left(T_{n}\right)$ of the $n$-th partial sums of the Faber expansion with respect to $K$ and therefore, in particular, in the case of $K=\boldsymbol{\Delta}_{r}$ for the Taylor sections $S_{n}$ and in the case $K=[-1,1]$ for the Tschebyscheff sections.

For a finite set $M \subset \mathbb{N}$ we define

$$
\Pi_{M}:=\left\{\sum_{\nu \in M} a_{\nu} z^{\nu}: a_{\nu} \in \mathbb{C} \text { for } \nu \in M\right\},
$$

i.e. $\Pi_{M}$ is the set of polynomials with powers only in $M$. (Note that always $Q(0)=0$ for $Q \in \Pi_{M}$.) If $Q \in \Pi_{M}$, then, by definition (15), we have $\varphi_{n}=e^{-R_{n}}$ where $R_{n} \in \Pi_{M}$ for all $n \in \mathbb{N}$. Therefore, the approximations of $f$ obtained by the $R G$-method are of the form

$$
\varphi_{n}^{-1} \cdot P_{n}=e^{R_{n}} \cdot P_{n} \quad \text { with } R_{n} \in \Pi_{M} \text { and } P_{n} \in \Pi_{n} .
$$

Since the effort for the evaluation of the factor $e^{R_{n}}$ does not increase with $n$ we may regard $\varphi_{n}^{-1} \cdot P_{n}$ as a "near polynomial approximation" of $f$. Theorems 1 and 5 show that, if $\tau(Q)<\tau$, we get a (geometric) acceleration factor $(\tau(Q) / \tau)^{n / \rho}$ if we approximate $f \varphi_{n}$ instead of $f$ by a polynomial sequence as in Theorem 5 . The "cost" for that is an additional multiplication by $\varphi_{n}^{-1}=e^{R_{n}}$.

\section{The polynomial $Q$}

We turn to the question of how to choose an appropriate polynomial $Q$ in order to apply the above $R G$-method in an efficient way. In view of the investigations made in Section 2 it is natural to consider the following problem:

Choose $Q_{M} \in \Pi_{M}$ such that

$$
\max _{\vartheta}\left(h_{f}(\vartheta)-\operatorname{Re} Q_{M}\left(e^{i \vartheta}\right)\right)=\min _{Q \in \Pi_{M}} \max _{\vartheta}\left(h_{f}(\vartheta)-\operatorname{Re} Q\left(e^{i \vartheta}\right)\right)
$$


(or at least a polynomial $Q \in \Pi_{M}$ "near" $Q_{M}$ ). This is a kind of one-sided Tschebyscheff approximation of the (continuous and $2 \pi$-periodic) function $h_{f}$ by trigonometric polynomials without constant term. The problem may be viewed as a semi-infinite optimization problem and has therefore in particular always a solution (cf. [15], Chapter 3). In our later applications it is in fact possible to solve the problem analytically. A method for solving (20) numerically is described in [21].

For the important case $M=\{1, \ldots, m\}$ we put

$$
Q_{m}:=Q_{\{1, \ldots, m\}}
$$

and obtain the following estimate.

Theorem 6. With the notations used above we have

$$
\underline{\tau} \leq \tau\left(Q_{m}\right) \leq \underline{\tau}+2 \varepsilon_{m}\left(h_{f}\right)
$$

where $\varepsilon_{m}\left(h_{f}\right)$ denotes the error of best approximation of $h_{f}$ by trigonometric polynomials of degree $\leq m$. This implies in particular

$$
\tau\left(Q_{m}\right) \rightarrow \underline{\tau} \quad(m \rightarrow \infty) .
$$

Proof. Let $t_{m}$ denote the best approximating trigonometric polynomial of degree $\leq m$ to the function $h_{f}$ on $[-\pi, \pi]$. If $a_{0}^{(m)} / 2$ is the constant term of $t_{m}$, that is

$$
\frac{a_{0}^{(m)}}{2}=\frac{1}{2 \pi} \int_{-\pi}^{\pi} t_{m}(\vartheta) d \vartheta
$$

then

$$
\frac{\left|a_{0}^{(m)}\right|}{2}=\frac{1}{2 \pi}\left|\int_{-\pi}^{\pi} h_{f}(\vartheta) d \vartheta+\int_{-\pi}^{\pi}\left(t_{m}(\vartheta)-h_{f}(\vartheta)\right) d \vartheta\right| \leq \underline{\tau}+\varepsilon_{m}\left(h_{f}\right),
$$

and therefore

$$
\max _{\vartheta}\left[h_{f}(\vartheta)-\left(t_{m}(\vartheta)-a_{0}^{(m)} / 2\right)\right] \leq \underline{\tau}+2 \varepsilon_{m}\left(h_{f}\right) .
$$

Since $t_{m}-a_{0}^{(m)} / 2$ is the real part of a polynomial in $e^{i \vartheta}$ of degree $\leq m$ without constant term, the assertion is proved.

Remark. 1. In many interesting cases $h_{f}$ is a "trigonometric spline" of order $\rho$, which means that there exists a partition $\vartheta_{0}<\vartheta_{1}<\ldots<\vartheta_{N}=\vartheta_{0}+2 \pi$ of $\left[\vartheta_{0}, \vartheta_{0}+2 \pi\right]$ and constants $a_{j}, b_{j}, j=1, \ldots, N$, such that

$$
h_{f}(\vartheta)=a_{j} \cos (\rho \vartheta)+b_{j} \sin (\rho \vartheta)
$$

for $\vartheta \in\left[\vartheta_{j-1}, \vartheta_{j}\right]$ and $j \in\{1, \ldots, N\}$. In this case, $h_{f}$ satisfies a Lipschitz condition of the form

$$
\left|h_{f}(\vartheta)-h_{f}(\tilde{\vartheta})\right| \leq c|\vartheta-\tilde{\vartheta}| \quad(\vartheta, \tilde{\vartheta} \in[-\pi, \pi])
$$

where $c:=\rho \cdot \max \left\{\left|a_{1}\right|, \ldots,\left|a_{N}\right|,\left|b_{1}\right|, \ldots,\left|b_{N}\right|\right\}$. Therefore, by an improved version of Jackson's theorem,

$$
\varepsilon_{m}\left(h_{f}\right) \leq \frac{\pi c}{2(m+1)} \quad(m \in \mathbb{N})
$$

(see for example [3], p. 143), and thus

$$
\tau\left(Q_{m}\right) \leq \underline{\tau}+\frac{\pi c}{m+1} \quad(m \in \mathbb{N}) .
$$


2. A particular simple but, nevertheless, for our later applications very interesting special case is

$$
\rho \in \mathbb{N} \text { and } M=\{\rho\} .
$$

In this case we have

$$
Q_{\rho}(z)=-a z^{\rho}
$$

with some constant $a=a\left(h_{f}\right)$ and therefore by (14) and (15)

$$
\varphi_{n}(z)=e^{-Q_{\rho}(z)} \quad(n \in \mathbb{N}),
$$

i.e., $\varphi_{n}$ is independent of $n$. For $\rho=1$, that is, $f$ is a function of exponential type $\tau$, and in the special case $K=\boldsymbol{\Delta}$, we note that for $P_{n}\left(f e^{-Q_{1}}\right)=S_{n}\left(f e^{-Q_{1}}\right)$, where $S_{n}$ denotes the $n$-th Taylor section, the $R G$-method coincides with the method introduced by Gabutti and Lyness in [8] (see also [7]). Observe, however, that in the paper [8], the computation of $a$ does not depend on $h_{f}$, but the singularities of the Borel transform of $f$.

\section{The POLYNomials $P_{n}$}

Assume that we have found a polynomial $Q$ such that $\tau>\tau(Q) \approx \underline{\tau}$. The question now is how to choose the approximating polynomial $P_{n}=P_{n}\left(f \varphi_{n}, K\right) \in$ $\Pi_{n}$ of $f \varphi_{n}$ on $K$. Since the polynomial $Q$ does only depend on $h_{f}$, we had so far no need to look on our compact set $K$ on which we want to approximate $f$. This set $K$ now plays an important role in order to choose $P_{n}$. Of course, concerning speed of approximation, the best possible choice is given by the sequence $P_{n}^{*}=P_{n}^{*}\left(f \varphi_{n}, K\right)$ of best approximating polynomials of $f \varphi_{n}$ with respect to $K$. However, in the most interesting cases of $K$ being a disk or an interval, also more explicit polynomial approximants are known, which are essentially as good as the polynomial best approximations.

It is worth while to be noted at this place that, since

$$
L_{n}\left(f \varphi_{n}\right)=L_{n}\left(L_{n}(f) L_{n}\left(\varphi_{n}\right)\right)
$$

for the polynomial interpolant of degree $\leq n$ in an arbitrary system of nodes $\left(z_{k}^{(n)}\right)$, the computation of $L_{n}\left(f \varphi_{n}\right)$ does not require more information about $f$ than the computation of $L_{n}(f)$, namely, the values of $f$ (and, in the case of multiple nodes, derivatives of $f$ ) at the nodes $\left(z_{k}^{(n)}\right)$.

1. The case $K=\boldsymbol{\Delta}_{r}$. Let $g$ be holomorphic in $\boldsymbol{\Delta}_{r}$ and let

$$
g(z)=\sum_{\nu=0}^{\infty} g_{\nu} z^{\nu} \quad\left(z \in \boldsymbol{\Delta}_{r}\right)
$$

be the Taylor expansion of $g$ around the origin. In the case $K=\boldsymbol{\Delta}_{r}$, the Taylor sections

$$
S_{n}(g)(z)=\sum_{\nu=0}^{n} g_{\nu} z^{\nu} \quad(z \in \mathbb{C})
$$

represent the interpolation polynomials of degree $\leq n$ to $g$ in the equidistributed nodes $z_{k}^{(n)}=0$ for $k=0, \ldots, n$ as well as the $n$-th partial sum of the Faber expansion with respect to $K$ of $g$. Since $c\left(\boldsymbol{\Delta}_{r}\right)=r$, by Theorem 5 we have

$$
\limsup _{n \rightarrow \infty} n^{1 / \rho}\left\|f-\varphi_{n}^{-1} S_{n}\left(f \varphi_{n}\right)\right\|_{\boldsymbol{\Delta}_{r}}^{1 / n} \leq r(e \rho \tau(Q))^{1 / \rho} .
$$


Another system of equidistributed nodes $\left(z_{k}^{(n)}\right)$ for $K=\boldsymbol{\Delta}_{r}$, where $r \geq 1$, consists of the $(n+1)$ st roots of unity

$$
z_{k}^{(n)}=e^{2 \pi i k /(n+1)} \quad\left(k=0, \ldots, n, n \in \mathbb{N}_{0}\right)
$$

([9], Chapter II).

In our numerical examples we will only consider (for $K=\boldsymbol{\Delta}_{r}$ ) the Taylor sections $S_{n}$, since they converge practically as fast as the best approximating polynomials (cf. for example [13]) on every $\boldsymbol{\Delta}_{r}$. If $Q(z)=\sum_{\nu \in M} a_{\nu} z^{\nu}$ for some $M \subset \mathbb{N}$ and if $\left(r_{n}\right)$ and $\left(\varphi_{n}\right)$ are given by (14) and (15), then the Taylor coefficients $\varphi_{k, n}=\varphi_{n}^{(k)}(0) / k$ ! of

$$
\varphi_{n}(z)=\exp \left(-r_{n}^{\rho} Q\left(z / r_{n}\right)\right)=\prod_{\nu \in M} \exp \left(-a_{\nu} z^{\nu} r_{n}^{\rho-\nu}\right)
$$

may be computed by repeated Cauchy product (i.e. by repeated discrete convolution) from the Taylor coefficients of $\exp \left(-a_{\nu} z^{\nu} r_{n}^{\rho-\nu}\right)$. Now, if the Taylor coefficients $f_{k}=f^{(k)}(0) / k$ ! of $f$ for $k=0, \ldots, n$ are known, one more Cauchy product gives

$$
S_{n}\left(f \varphi_{n}\right)(z)=\sum_{\nu=0}^{n} z^{\nu}\left(\sum_{k=0}^{\nu} f_{k} \varphi_{\nu-k, n}\right) .
$$

However, if the Taylor coefficients $f_{0}, \ldots, f_{n}$ of $f$ are computed numerically by a quadrature forumula or by Fast Fourier Transform (cf. [19]), then it is convenient to compute also the coefficients $\sum_{k=0}^{\nu} f_{k} \varphi_{\nu-k, n}$ of $f \varphi_{n}$ directly by a quadrature formula or FFT.

2. The case $K=[a, b]$. As is well-known, in the case $K=[-1,1]$ systems of equidistributed nodes are for example the zeros of the Tschebyscheff polynomials

$$
z_{k}^{(n)}=\cos \left(\frac{(2 k+1) \pi}{2(n+1)}\right), \quad k=0, \ldots, n,
$$

or the Fejér nodes given by

$$
z_{k}^{(n)}=\cos \left(\frac{2 k \pi}{n+1}\right), \quad k=0, \ldots, n
$$

(see for example [9], Chapter II). Since in the second case $z_{k}^{(n)}=z_{n-k+1}^{(n)}$ for $k=$ $1, \ldots, n$, we have interpolation of $f$ and $f^{\prime}$ in these nodes.

As remarked in Section 1, the Faber polynomials for $K=[-1,1]$ coincide with the (normalized) Tschebyscheff polynomials, more precisely,

$$
F_{n}(x)= \begin{cases}2 \cdot \cos (n \arccos x), & \text { if } n=1,2, \ldots, \\ 1, & \text { if } n=0,\end{cases}
$$

for $x \in[-1,1]$ (see for example [20], p. $110_{3}$ ), and the $n$-th partial sum $T_{n}$ of the Faber expansion equals the $n$-th partial sum of the Tschebyscheff expansion. Since $c([-1,1])=1 / 2$, Theorem 5 gives

$$
\limsup _{n \rightarrow \infty} n^{1 / \rho}\left\|f-\varphi_{n}^{-1} T_{n}\left(f \varphi_{n}\right)\right\|_{[-1,1]}^{1 / n} \leq \frac{1}{2}(e \rho \tau(Q))^{1 / \rho} .
$$

Thus we see that the smaller capacity of $K=[-1,1]$ compared to $K=\boldsymbol{\Delta}$ causes an acceleration factor of $(1 / 2)^{n}$ if $f$ is approximated by $\varphi_{n}^{-1} T_{n}\left(f \varphi_{n}\right)$ instead of $\varphi_{n}^{-1} S_{n}\left(f \varphi_{n}\right)$ on $[-1,1]$. 
The case of an arbitrary interval $K=[a, b]$ with $a, b \in \mathbb{C}$ may be reduced to the case $K=[-1,1]$ by a simple linear transformation, so that this case is essentially included above. In particular, for a function $g$ holomorphic on $[a, b]$ the $n$-th Faber section $T_{n}(g)=T_{n,[a, b]}(g)$ with respect to $[a, b]$ is given by

$$
T_{n,[a, b]}(g)(w)=T_{n,[-1,1]}(\tilde{g})\left(\frac{2}{b-a} w-\frac{b+a}{b-a}\right),
$$

where

$$
\tilde{g}(z):=g\left(\frac{b-a}{2} z+\frac{a+b}{2}\right) .
$$

As in the standard case $[a, b]=[-1,1]$ we denote $T_{n,[a, b]}(g)$ as $n$-th Tschebyscheff section of $g$ (with respect to $[a, b]$ ).

Since the partial sums $T_{n}$ of the Tschebyscheff expansion converge practically as fast as the best approximations on $K=[a, b]$ (see for example [25], p. 134), and since, on the other hand, they have computational advantages (cf. [26]), we will restrict ourselves in the numerical examples for $K=[a, b]$ to the Tschebyscheff expansion. In most cases, Tschebyscheff coefficients are computed numerically by some quadrature formula ([25], p. $148 \mathrm{ff}$ ) or by Fast Fourier Transform (cf. [12]). Of course, the same methods may be used to compute the coefficients of $f \varphi_{n}$ numerically.

3. More general $K$. More general compact sets $K$ (having simply connected complement $\hat{\mathbb{C}} \backslash K$ ) may be handled similar to the above case of $K=[a, b]$ by choosing the $n$-th partial sum $T_{n}$ of the Faber expansion instead of the $n$-th Tschebyscheff section. An efficient method for the numerical evaluation of $T_{n}$ is described in [5]. Moreover, in [4] and [10] explicit expressions for the Faber polynomials $F_{n, K}$ in the cases of $K$ being a circular or an annular sector are given.

\section{NumeriCAL EXAMPLES}

In our numerical examples we restrict ourselves to the compact sets $K$ being the most simple and the most important ones, namely closed circles $\boldsymbol{\Delta}_{r}$ and compact (real) intervals. Thereby we express the numerical results in terms of (an approximation of) the function sd defined for a compact set $K \subset \mathbb{C}$, an entire function $f$ of order $\rho$ and an approximation $\tilde{f}$ of $f$ by

$$
\operatorname{sd}(K):=\operatorname{sd}(K ; f, \tilde{f}):=\inf _{z \in K}\left(-\log _{10}|f(z)-\tilde{f}(z)|+h_{f}(\arg z)|z|^{\rho} / \log (10)\right) .
$$

This function may be viewed as an approximation of the number of significant decimal digits achieved by the approximation $\tilde{f}$ of $f$ on $K$ except for neighbourhoods of the zeros of $f$. We hereby replace the more accurate relative error $-\log _{10}|(f(z)-\tilde{f}(z)) / f(z)|$ by

$$
-\log _{10}\left|\frac{f(z)-\tilde{f}(z)}{e^{h_{f}(\arg z)|z|^{\rho}}}\right|=-\log _{10}|f(z)-\tilde{f}(z)|+h_{f}(\arg z)|z|^{\rho} / \log (10),
$$

since in this way we can avoid problems obviously occurring near the zeros of $f$. On the other hand, $\operatorname{sd}(K)$ is a more suitable approximation of the number of significant decimal digits than the absolute error $-\log _{10}\|f-\tilde{f}\|_{K}$, since the error in $\operatorname{sd}(K)$ is normalized in sectors where $f$ grows or decreases exponentially fast. 
The numerical calculations were carried out in double precision Fortran, which gives a maximal accuracy of 16 decimal digits. As "exact" functions $f$ we used certain high degree Taylor sections.

1. Confluent hypergeometric functions. In our first numerical example we suppose $f$ to be a confluent hypergeometric function

$$
K(a, c ; z):=\sum_{\nu=0}^{\infty} \frac{(a)_{\nu} z^{\nu}}{(c)_{\nu} \nu !}
$$

where $(a)_{0}:=1$ and

$$
(a)_{\nu}:=a(a+1) \cdots(a+\nu-1) \quad(\nu \in \mathbb{N})
$$

is Pochhammer's notation. The confluent hypergeometric functions are of order 1 , type 1 , and have (for $a \neq c$ ) indicator function

$$
h(\vartheta)=\left\{\begin{array}{ll}
\cos (\vartheta), & \text { if }|\vartheta| \leq \pi / 2 \\
0, & \text { else }
\end{array} \quad \text { with } \underline{\tau}=\frac{1}{\pi} .\right.
$$

We apply the $R G$-method for the two cases $M=\{1\}$ and $M=\{1,2\}$.

If $M=\{1\}$, then the minimax problem (20) has the solution

$$
Q_{1}(z)=z / 2 \text { and } \tau\left(Q_{1}\right)=1 / 2 .
$$

Since $\rho=1$ and $M=\{1\}$, the functions $\varphi_{n}=e^{-Q_{1}}$ given by (15) are independent of $n$ and the power series of $f e^{-Q_{1}}$ is given by

$$
f(z) e^{-Q_{1}(z)}=K(a, c ; z) e^{-z / 2}=\sum_{\nu=0}^{\infty} z^{\nu}\left(\frac{(-1)^{\nu}}{2^{\nu} \nu !} \sum_{k=0}^{\nu}\left(\begin{array}{l}
\nu \\
k
\end{array}\right)(-2)^{k} \frac{(a)_{k}}{(c)_{k}}\right) .
$$

If we replace for $K=\boldsymbol{\Delta}_{r}$ the Taylor sections $S_{n}(f)$ by $e^{z / 2} S_{n}\left(f e^{-Q_{1}}\right)(z)$ or if we replace for $K=[a, b]$ the Tschebyscheff sections $T_{n}(f)$ by $e^{z / 2} T_{n}\left(f e^{-Q_{1}}\right)(z)$ then, according to Theorems 1 and 5, we get an (asymptotic) acceleration factor of

$$
\left(\tau\left(Q_{1}\right) / \tau\right)^{n}=(1 / 2)^{n} .
$$

As noted above, for $M=\{1\}$ the $R G$-method coincides with the method introduced by Gabutti and Lyness [8] for the case of the Taylor sections. They find the polynomial $Q_{1}$ by "Symmetrization" of the indicator diagram of $f$, which is $[0,1]$ here.

Besides the use of Taylor sections, an efficient method for evaluating $K(1, c ; z)$ for complex $z$ is given by the continued fractions expansion

$$
K(1, c ; z)=\frac{a_{1}}{1+} \frac{a_{2} z}{1+} \frac{a_{3} z}{1+} \ldots
$$

where $a_{1}=1, a_{2}=-1 / c$ and

$$
a_{2 k+1}=\frac{k}{(c+2 k-2)(c+2 k-1)}, \quad a_{2 k+2}=-\frac{c+k-1}{(c+2 k-1)(c+2 k)}
$$

(see for example [16]). As is well-known, the approximants of (26) form a stair step sequence in the Padé table of $K(1, c ; z)$, that is, the $n$-th approximant

$$
f_{n}(z)=\frac{a_{1}}{1+} \frac{a_{2} z}{1+} \ldots \frac{a_{n} z}{1}
$$


coincides with the $\left(\left[\frac{n-1}{2}\right],\left[\frac{n}{2}\right]\right)$-degree Padé approximation of $K(1, c ; \cdot)$. As may be found in [6], the asymptotic rate of convergence of the $(m, m)$-degree Padé approximations $R_{m}$ of $K(1, c ; \cdot)$ is given by

$$
\limsup _{m \rightarrow \infty}(2 m)\left\|K(1, c ; \cdot)-R_{m}\right\|_{\Delta_{r}}^{1 / 2 m}=e r / 2 .
$$

Thus, for $n=2 m+1$ we find

$$
\limsup _{n \rightarrow \infty} n\left\|K(1, c ; \cdot)-f_{n}\right\|_{\Delta_{r}}^{1 / n}=e r / 2 .
$$

(Actually, the same is true for arbitrary $n$.) This is the same rate as for the Taylor sections $S_{n}\left(f \varphi^{(1)}\right)$ for $f(z)=K(1, c ; z)$. Since the sum of the numerator and denominator polynomial degree of $f_{n}$ is (essentially) $n$, once the coefficients are stored, the effort for evaluating $f_{n}$ and $S_{n}\left(f e^{-Q_{1}}\right)$ is essentially the same.

In the case $M=\{1,2\}$ the minimax problem (20) is approximately solved by

$$
Q(z)=\frac{z}{2}+\frac{z^{2}}{4 \sqrt{2}}
$$

and this solution also seems to be exact (added in proof: it is exact, as was told to me by K. Petras). We find

$$
\tau(Q)=\frac{1}{2 \sqrt{2}}=0.35 \ldots
$$

which, according to (14) and (15), yields

$$
\varphi_{n}(z)=\varphi_{n, Q}(z)=\exp \left(-\frac{z}{2}-\frac{z^{2}}{16 n}\right) .
$$

Thus, if we use the polynomial $Q$ instead of $Q_{1}$, we achieve a further acceleration factor of $\left(\tau(Q) / \tau\left(Q_{1}\right)\right)^{n}=(1 / \sqrt{2})^{n}$. Moreover, since $\tau(Q) / \underline{\tau}=\pi /(2 \sqrt{2}) \approx 1.1$ does not differ essentially from 1 , it is of little interest to consider any further case of $M$.

In our test example we consider the (besides the exponential function) most prominent member $K\left(\frac{1}{2}, \frac{3}{2} ; \cdot\right)$ of the class of confluent hypergeometric functions, which is the essential ingredient of the error function by

$$
\operatorname{erf}(z)=\frac{2 z}{\sqrt{\pi}} K\left(\frac{1}{2}, \frac{3}{2} ;-z^{2}\right) .
$$

Figure $1 \operatorname{shows} \operatorname{sd}\left(\boldsymbol{\Delta}_{r}\right)$ as a function of $r$ for $\tilde{f}(z)=e^{z / 2} S_{12}\left(f e^{-Q_{1}}\right)(z)(R G 1)$ and $\tilde{f}=\varphi_{12}^{-1} S_{12}\left(f \varphi_{12}\right)(R G 2)$ as well as for $\tilde{f}=S_{12}(f)$, the 12-th Taylor section of $f$ $(T S)$.

We have added $\operatorname{sd}\left(\boldsymbol{\Delta}_{r}\right)$ also for $\tilde{f}(z)=e^{z} f_{12}(-z)$, where $f_{12}$ is the continued fractions approximant (27) of $K\left(1, \frac{3}{2} ; z\right)(C F)$. (By Kummer's first identity we have $K\left(\frac{1}{2}, \frac{3}{2}, z\right)=e^{z} K\left(1, \frac{3}{2} ;-z\right)$.)

Since $(0.5)^{12} \approx 10^{-3.6}$ and $(0.35)^{12} \approx 10^{-5.5}$, we can expect $3-4$ extra significant decimal digits for $R G 1$ and 5-6 extra significant decimal digits for $R G 2$. Figure 1 shows that these acceleration factors are actually achieved. As we have seen above, the asymptotic rate of convergence for $R G 1$ is the same as for $C F$, but actually for small values of $n$ the method $R G 1$ turns out to be somewhat more accurate.

Table 1 shows the values of $\operatorname{sd}(K)$ for several intervals $K$ for $\tilde{f}(z)=$ $e^{z / 2} T_{10}\left(f e^{-Q_{1}}\right)(z)(R G 1)$ and $\tilde{f}=\varphi_{10}^{-1} T_{10}\left(f \varphi_{10}\right)(R G 2)$ as well as for the Tschebyscheff section $T_{10}(f)$ of $f(T S C H S)$. 


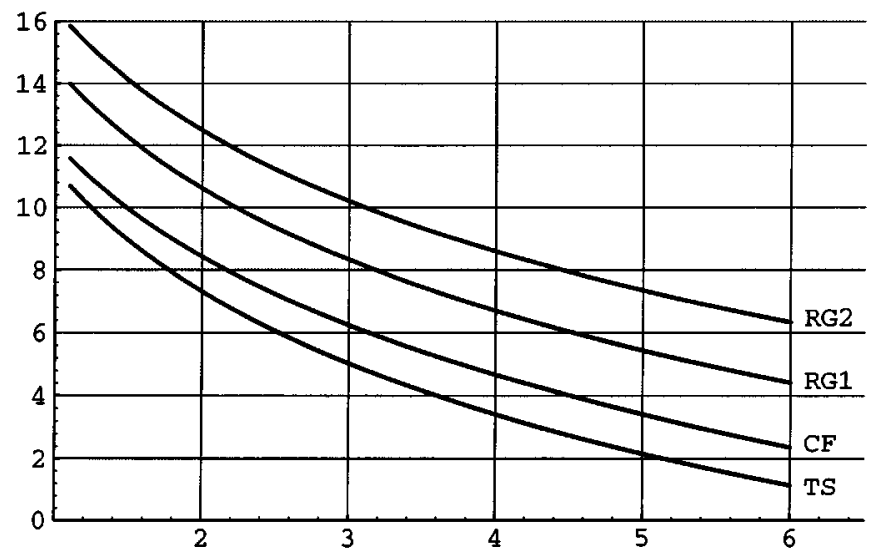

Figure 1. $\operatorname{sd}\left(\boldsymbol{\Delta}_{r}\right)$ for $f=K(1 / 2,3 / 2 ; \cdot)$

TABLE $1 . \operatorname{sd}(K)$ for $f=K(1 / 2,3 / 2 ; \cdot)$

\begin{tabular}{|l|c|c|c|}
\hline Interval & TSCHS & $R G 1$ & $R G 2$ \\
\hline \hline$K=[-2,2]$ & 8.6 & 11.4 & 12.9 \\
\hline$K=[0,4]$ & 7.7 & 11.9 & 12.7 \\
\hline$K=[-4,0]$ & 9.4 & 10.9 & 12.8 \\
\hline
\end{tabular}

Since $(0.5)^{10} \approx 10^{-3}$ and $(0.35)^{10} \approx 10^{-4.5}$, we can expect about 3 extra decimal digits in the $R G 1$ case and about $4-5$ extra decimal digits in the $R G 2$ case. For $K=[-2,2]$, this acceleration is realized. We find that $T_{n}(f)$ behaves for small $n$ on $K=[-4,0]$ better than theoretically expected and on $K=[0,4]$ not as good as theoretically expected. Thus, the $R G$-method is somewhat more effective in the case $K=[0,4]$.

It has to be noted that besides the computation of a polynomial (of degree 12 or 10 in our examples), the $R G 1$ - and the $R G 2$-method, as the method $C F$, require an additional evaluation of the exponential function. However, since in most algorithms for the complex error function the so-called Faddeeva function $\omega$ is computed instead of erf (see for example [11], [23], [29]), one has to multiply by an exponential term anyway, so that there may be no extra effort.

For example in the algorithm given in [23], which seems to be the state of the art (cf. [29]), $\omega$ is computed by truncation of the power series in

$$
\omega(z)=e^{-z^{2}}\left(1+\frac{2 i}{\sqrt{\pi}} \sum_{\nu=0}^{\infty} \frac{z^{2 \nu+1}}{(2 \nu+1) \nu !}\right)=e^{-z^{2}}\left(1+\frac{2 i z}{\sqrt{\pi}} K\left(\frac{1}{2}, \frac{3}{2} ; z^{2}\right)\right)
$$

for $z$ in a certain neighbourhood of the origin (and in the first quadrant). The extra evaluation of the exponential function in the case of the $R G 1$-method may be avoided by computing with $f(w) e^{-Q_{1}(w)}=K\left(\frac{1}{2}, \frac{3}{2} ; w\right) e^{-w / 2}$ the approximation

$$
\left(e^{-z^{2} / 2}\right)^{2}+\frac{2 i z e^{-z^{2} / 2}}{\sqrt{\pi}} S_{n}\left(f e^{-Q_{1}}\right)\left(z^{2}\right)
$$


of $\omega(z)$. So, storing the coefficients of $f e^{-Q_{1}}$ (see (25)) and evaluating $S_{n}\left(f e^{-Q_{1}}\right)\left(z^{2}\right)$ for example by Horner's algorithm may be an interesting alternative in the algorithm given in [23]. The same could be true for the $R G 2$-method, where the higher speed of the polynomial approximations stands against one extra evaluation of the exponential function and the disadvantage that $f \varphi_{n}$ depends on $n$.

2. Bessel functions. In our second numerical example we are concerned with the Bessel functions $J_{\lambda}$ and $Y_{\lambda}$. A fundamental system of Bessel's differential equation is given by

$$
J_{\lambda}(z)=\left(\frac{z}{2}\right)^{\lambda} \sum_{\nu=0}^{\infty} \frac{\left(-z^{2}\right)^{\nu}}{\nu ! \Gamma(\lambda+\nu+1) 4^{\nu}}=\frac{1}{\Gamma(\lambda+1)}\left(\frac{z}{2}\right)^{\lambda} \sum_{\nu=0}^{\infty} \frac{\left(-z^{2}\right)^{\nu}}{(\lambda+1)_{\nu} \nu ! 4^{\nu}},
$$

the Bessel function of order $\lambda$ of first kind, and the Bessel function of order $\lambda$ of second kind $Y_{\lambda}$, defined by

$$
Y_{\lambda}(z)=\frac{J_{\lambda}(z) \cdot \cos (\lambda \pi)-J_{-\lambda}(z)}{\sin (\lambda \pi)}
$$

for $\lambda \notin \mathbb{Z}$. Thus, for the evaluation of $J_{\lambda}$ and $Y_{\lambda}$ in the case $\lambda \notin \mathbb{Z}$ it is essential to compute

$$
f_{\lambda}(z)={ }_{0} F_{1}\left(\lambda+1 ; \frac{z}{4}\right)=\sum_{\nu=0}^{\infty} \frac{z^{\nu}}{(\lambda+1)_{\nu} \nu ! 4^{\nu}} .
$$

The functions $f_{\lambda}$ are of order $\rho=1 / 2$ and type $\tau=1$ with indicator function

$$
h_{f_{\lambda}}(\vartheta)=h(\vartheta)=\cos (\vartheta / 2) .
$$

For $M=\{1\}$, the minimax problem (20) has the solution

$$
Q_{1}(z)=\frac{z}{2 \sqrt{2}} \quad \text { and } \quad \tau\left(Q_{1}\right)=\frac{1}{\sqrt{2}}
$$

as may be seen by some elementary calculus. According to Theorems 1 and 5 we achieve an acceleration factor of

$$
\tau\left(Q_{1}\right)^{2 n}=(1 / 2)^{n}
$$

if we replace the Taylor sections $S_{n}\left(f_{\lambda}\right)$ by $\varphi_{n}^{-1} S_{n}\left(f_{\lambda} \varphi_{n}\right)$ or if we replace the Tschebyscheff sections $T_{n}\left(f_{\lambda}\right)$ by $\varphi_{n}^{-1} T_{n}\left(f_{\lambda} \varphi_{n}\right)$. By (14) and (15) we obtain

$$
\varphi_{n}(z)=\exp \left(-\frac{z}{8 n}\right) \text {. }
$$

Therefore, the Taylor sections $S_{n}\left(f_{\lambda} \varphi_{n}\right)$ may be explicitly computed as

$$
S_{n}\left(f_{\lambda} \varphi_{n}\right)(z)=\sum_{\nu=0}^{n} z^{\nu}\left(\frac{(-1)^{\nu}}{\nu !(8 n)^{\nu}} \sum_{k=0}^{\nu}\left(\begin{array}{l}
\nu \\
k
\end{array}\right) \frac{(-1)^{k}(8 n)^{k}}{(\lambda+1)_{k}}\right) .
$$

Figure $2 \operatorname{shows} \operatorname{sd}\left(\boldsymbol{\Delta}_{r}\right)$ as a function of $r$ for

$$
f_{1 / 2}(z)={ }_{0} F_{1}\left(\frac{3}{2} ; \frac{z}{4}\right)=\frac{\sinh (\sqrt{z})}{\sqrt{z}}
$$

and $\tilde{f}=\varphi_{10}^{-1} S_{10}\left(f_{1 / 2} \varphi_{10}\right)(R G)$ as well as $\tilde{f}=S_{10}\left(f_{1 / 2}\right)(T S)$.

Since $(0.5)^{10} \approx 10^{-3}$, we can expect about 3 extra significant decimal digits for $R G$ compared to $T S$. The numerical results show that the (asymptotic) acceleration factor is (at least) realized even for small $n$. It has to be remarked that, as in the first example, in the $R G$-case besides the evaluation of a polynomial (here of degree 10) an extra calculation of $\varphi_{n}^{-1}(z)=\exp (z / 8 n)$ has to be carried out. However, 


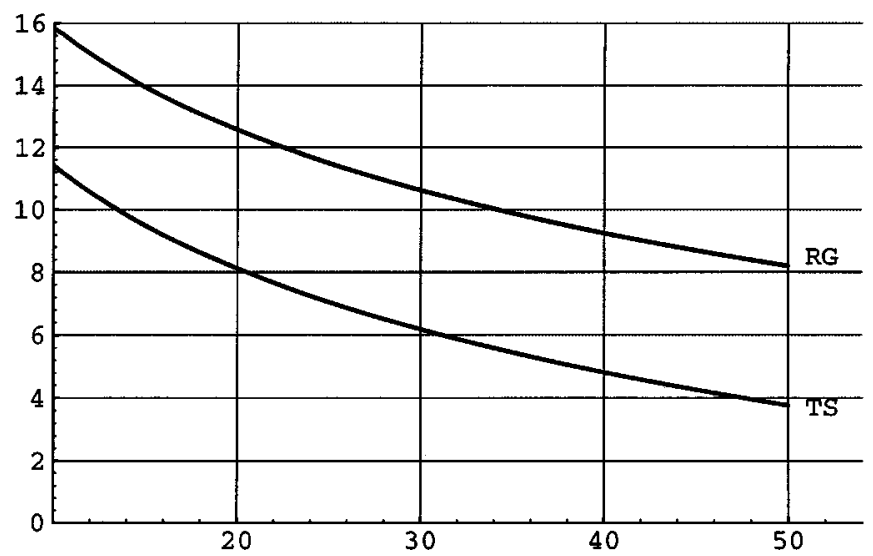

Figure 2. $\operatorname{sd}\left(\boldsymbol{\Delta}_{r}\right)$ for $f_{1 / 2}$

since the computation of $J_{\lambda}$ requires also the calculation of the factor $z^{\lambda}$, which in the case $\lambda \in \mathbb{C} \backslash \mathbb{Z}$ is computed as $\exp (\lambda \log z)$, the factor $\exp (z / 8 n)$ may be integrated into this evaluation of the exponential.

Of course, a similar argumentation applies to the case of an interval and the corresponding Tschebyscheff sections $T_{n}$. On this "real-variable case" we will focus our attention now.

For $\lambda \in \mathbb{N}_{0}$, the Bessel functions of second kind are given by

$$
\begin{aligned}
Y_{\lambda}(z) & =\frac{2}{\pi} J_{\lambda}(z)\left(\gamma+\log \frac{z}{2}\right) \\
& -\frac{1}{\pi}\left(\frac{z}{2}\right)^{\lambda} \frac{1}{\Gamma(\lambda+1)}\left[\left(\psi_{0}+\psi_{\lambda}\right) * f_{\lambda}\right]\left(-z^{2}\right) \\
& -\frac{1}{\pi}\left(\frac{z}{2}\right)^{-\lambda} \sum_{k=0}^{\lambda-1} \frac{(\lambda-k-1)}{k !}\left(\frac{z}{2}\right)^{2 k} .
\end{aligned}
$$

Here, $\gamma$ is Euler's constant and

$$
\psi_{m}(z):=\sum_{k=0}^{\infty} z^{k}\left(\sum_{\ell=1}^{m+k} \frac{1}{\ell}\right)
$$

where $\sum_{\phi}:=0$, and $*$ denotes the Hadamard product of power series (see for example [14], 9.7). Thus, the evaluation of $Y_{\lambda}$ for $\lambda \in \mathbb{N}_{0}$ requires a further essential computation of

$$
g_{\lambda}:=\left(\psi_{0}+\psi_{\lambda}\right) * f_{\lambda},
$$

for which it may also be shown that

$$
h_{g_{\lambda}}=h_{f_{\lambda}}=h .
$$

In many applications (for example series expansions in Bessel functions) one needs arrays $\left\{J_{0}(z), \ldots, J_{N}(z)\right\}$ and $\left\{Y_{0}(z), \ldots Y_{N}(z)\right\}$ of Bessel functions of first and second kind. Such arrays are usually computed by recurrence relations. Since the 2 -term recurrence in ascending order

$$
J_{\lambda+1}(z)=\frac{2 \lambda}{z} J_{\lambda}(z)-J_{\lambda-1}(z)
$$


is in general numerically unstable, one applies recurrence in descending order for the computation of Bessel functions of the first kind $J_{\lambda}$. This leads to the so-called Miller algorithm (cf. [28]). On the contrary, Bessel functions of the second kind $Y_{\lambda}$ may be computed stably by using the recurrence formula in ascending order

$$
Y_{\lambda+1}(z)=\frac{2 \lambda}{z} \cdot Y_{\lambda}(z)-Y_{\lambda-1}(z)
$$

Thus it is of fundamental importance to find effective methods for the calculation of $Y_{0}$ and $Y_{1}$. As we have seen above, the functions $Y_{0}$ and $Y_{1}$ are essentially built up from the four entire functions of order $1 / 2$

$$
f_{0}={ }_{0} F_{1}\left(1 ; \frac{\dot{-}}{4}\right) \quad, \quad f_{1}={ }_{0} F_{1}\left(2 ; \frac{\dot{-}}{4}\right)
$$

and

$$
g_{0}=2 \psi_{0} * f_{0} \quad, \quad g_{1}=\left(\psi_{0}+\psi_{1}\right) * f_{1}
$$

all having indicator function $h$. Therefore, if we apply the $R G$-method, one polynomial $Q$ applies to $f_{0}, f_{1}, g_{0}$ and $g_{1}$. As in the case $\lambda \in \mathbb{C} \backslash \mathbb{Z}$, we get for $M=\{1\}$

$$
Q_{1}(z)=\frac{z}{2 \sqrt{2}} \quad \text { and } \quad \tau\left(Q_{1}\right)=\frac{1}{\sqrt{2}} .
$$

In the numerical examples for the evaluation of $f_{0}, f_{1}, g_{0}$ and $g_{1}$ we restrict ourselves to the real variable case, namely $K$ is one of the intervals $K_{1}=[-50,0]$ and $K_{2}=[-100,0]$. Intervals on the negative half axis play the most important role since approxmations of $f_{0}, f_{1}, g_{0}$ and $g_{1}$ on the negative half axis are needed for the approximation of $Y_{0}$ and $Y_{1}$ on the positive real axis. In our examples of $K_{1}=[-50,0]$ and $K_{2}=[-100,0]$, respectively, the approximations of $f_{0}, f_{1}, g_{0}$ and $g_{1}$ yield approximations of $Y_{0}$ and $Y_{1}$ on $\tilde{K}_{1}=(0, \sqrt{50}]$ and $\tilde{K}_{2}=(0,10]$, respectively.

Tables $2-5$ show the approximations of the significant decimal digits $\operatorname{sd}\left(K_{1}\right)$ and $\operatorname{sd}\left(K_{2}\right)$ for the functions $f \in\left\{f_{0}, f_{1}, g_{0}, g_{1}\right\}$ and the approximants $T_{10}(f)(T S C H S)$ and $\varphi_{10}^{-1} T_{10}\left(f \varphi_{10}\right)$.

The numerical results show that the expected acceleration factor $(0.5)^{10} \approx 10^{-3}$ is actually achieved.

Besides the evaluation of a polynomial of degree 10 , the $R G$-method requires the computation of the exponential factor $\varphi_{10}^{-1}(z)=\exp (z / 80)$. However, the extra evaluation of the exponential function need only be carried out once for the approximation of $f_{0}, f_{1}, g_{0}$ and $g_{1}$.

Although we have here only considered the real-variable case, similar results may be obtained for $K=\boldsymbol{\Delta}_{r}$. Moreover, in [21] the RG-method is also applied to Bessel functions by using a sequence of multipliers $\left(\varphi_{n}\right)_{n}$ consisting of rational functions, and to the Airy function.

TABLE 2. $\operatorname{sd}(K)$ for $f_{0}$

\begin{tabular}{|l|c|c|}
\hline Interval & $T S C H S$ & $R G$ \\
\hline \hline$K_{1}$ & 9.7 & 12.7 \\
\hline$K_{2}$ & 6.6 & 9.3 \\
\hline
\end{tabular}


TABLE $3 \cdot \operatorname{sd}(K)$ for $f_{1}$

\begin{tabular}{|l|c|c|}
\hline Interval & $T S C H S$ & $R G$ \\
\hline \hline$K_{1}$ & 10.7 & 14.7 \\
\hline$K_{2}$ & 7.6 & 11.1 \\
\hline
\end{tabular}

TABLE 4. $\operatorname{sd}(K)$ for $g_{1}$

\begin{tabular}{|l|c|c|}
\hline Interval & $T S C H S$ & $R G$ \\
\hline \hline$K_{1}$ & 8.9 & 11.8 \\
\hline$K_{2}$ & 5.8 & 8.4 \\
\hline
\end{tabular}

TABLE $5 \cdot \operatorname{sd}(K)$ for $g_{1}$

\begin{tabular}{|l|c|c|}
\hline Interval & $T S C H S$ & $R G$ \\
\hline \hline$K_{1}$ & 10.0 & 13.7 \\
\hline$K_{2}$ & 6.9 & 10.0 \\
\hline
\end{tabular}

Finally, we mention the articles [30] and [22] where an alternative method for convergence acceleration of entire functions of finite order is described.

\section{REFERENCES}

1. Amos, D.E., A portable package for Bessel functions of a complex argument and nonnegative order, ACM Trans. Math. Softw., 12 (1986), 265-273 CMP 19:12

2. Berenstein, C.A., Gay, R., Complex analysis and special topics in harmonic analysis, Springer, New York, 1995. MR 96j:30001

3. Cheney, E.W., Approximation theory, McGraw-Hill, New York, 1966. MR 36:5568

4. Coleman, J.P., Myers, N.J., The Faber polynomials for annular sectors, Math. Comp., 64 (1995), 181-203. MR 95c:30006

5. Ellacott, S.W., Computation of Faber series with application to numerical polynomial approximation in the complex plane, Math. Comp., 40 (1983), 575-587. MR 84e:65021

6. Elliott, D., Truncation error in Padé approximations to certain functions, an alternative approach, Math. Comp., 21 (1967), 398-406. MR 37:3252

7. Gabutti, B., On two methods for accelerating convergence of series, Numer. Math., 43 (1984), 439-461. MR 85i:65005

8. Gabutti, B., Lyness, J.N., An acceleration method for the power series of entire functions of order 1, Math. Comp., 39 (1982), 587-597. MR 83j:65011

9. Gaier, D., Lectures on complex approximation, Birkhäuser, Boston, 1987. MR 88i:30059b

10. Gatermann, K., Hoffmann, Ch., Opfer, G., Faber polynomials on circular sectors, Math. Comp., 58 (1992), 241-253. MR 92h:30011

11. Gautschi, W., Efficient computation of the complex error function, SIAM J. Numer. Anal., 7 (1970), 187-198. MR 45:2889

12. Geddes, K.O., Near minimax polynomial approximation in an elliptical region, SIAM J. Numer. Anal., 15 (1978), 1225-1233. MR 80a:65062

13. Geddes, K.O., Mason, J.C., Polynomial approximation by projections on the unit circle, SIAM J. Numer. Anal., 12 (1975), 111-120. MR 51:1230

14. Henrici, P., Applied and computational complex analysis, Vol. II, Wiley, New York, 1991. MR 93b:30001 
15. Hettich, R., Zencke, P., Numerische Methoden der Approximation und semi-infiniten Optimierung, Teubner, Stuttgart, 1982. MR 84a:90069

16. Jones, W.B., Thron, W.F., On the computation of incomplete gamma functions in the complex domain, J. Comp. Appl. Math., 12 \& 13 (1985), 401-417. MR 87c:65023

17. Kövari, T., Pommerenke, Ch., On Faber polynomials and Faber expansions, Math. Z., 99 (1967), 193-206. MR 37:3013

18. Lewin, B.J., Distribution of zeros of entire functions, Amer. Math. Soc., 1964.

19. Lyness, J.N., Sande, G., Algorithm 413, Evaluation of normalized Taylor coefficients of an analytic function, Comm. ACM, 14 (1971), 669-675.

20. Markushevich, A.I., The theory of functions, 2nd ed., Chelsea, New York, 1977. MR 56:3258

21. Müller, J., Convergence acceleration of polynomial expansions for finite order entire functions, Habilitationsschrift, Trier 1995.

22. Müller, J., Convergence acceleration of Taylor sections for finite order entire functions, submitted.

23. Poppe; G.P.M., Wijers, C.M., More efficient computation of the complex error function, ACM Trans. Math. Softw., 16 (1990), 38-46. MR 91h:65068a

24. Rice, J.R., The degree of convergence for entire functions, Duke Math. J., 38 (1971), 429-440. MR 44:4217

25. Rivlin, T.J., The Chebyshev polynomials, Wiley, New York, 1974. MR 56:9142

26. Schonfelder, J.L., Special functions in the NAG library. In: Software for numerical mathematics, D.J. Evans (Editor), Academic Press, New York, 1974. MR 50:6095

27. Steinmetz, N., Exceptional values of solutions of linear differential equations, Math. Z., 201 (1989), 317-326. MR 90i:30044

28. Watanabe, T., Natori, M., Oguni, T., Mathematical software for the P.C. and work stations, North-Holland, Amsterdam, 1994. MR 95g:65008

29. Weideman, J.A.C., Computation of the complex error function, SIAM J. Numer. Anal., 31 (1994), 1497-1518. MR 95h:65012a

30. Wild, P., Accelerating the convergence of power series of certain entire functions, Numer. Math., 51 (1987), 583-595. MR 89a:65007

31. Winiarski, T., Approximation and interpolation of entire functions, Ann. Polon. Math. 23 (1970), 259-273. MR 42:7913

Fachbereich IV-Mathematik, Universität Trier, D-54286 Trier, Germany

E-mail address: jmueller@uni-trier.de 